\title{
The Role of Friction in the Static Equilibrium of a Fixed Ladder: Theoretical Analysis and Experimental Test
}

\author{
M. P. Silverman \\ Department of Physics, Trinity College, Hartford CT, USA \\ Email: mark.silverman@trincoll.edu
}

How to cite this paper: Silverman, M.P. (2018) The Role of Friction in the Static Equilibrium of a Fixed Ladder: Theoretical Analysis and Experimental Test. World Journal of Mechanics, 8, 445-463. https://doi.org/10.4236/wjm.2018.812032

Received: November 2, 2018

Accepted: December 27, 2018

Published: December 30, 2018

Copyright $\odot 2018$ by author(s) and Scientific Research Publishing Inc. This work is licensed under the Creative Commons Attribution International License (CC BY 4.0).

http://creativecommons.org/licenses/by/4.0/

\begin{abstract}
In a recent publication the author derived and experimentally tested several theoretical models, distinguished by different boundary conditions at the contacts with horizontal and vertical supports, that predicted the forces of reaction on a fixed (i.e. inextensible) ladder. This problem is statically indeterminate since there are 4 forces of reaction and only 3 equations of static equilibrium. The model that predicted the empirical reactions correctly used a law of static friction to complement the equations of static equilibrium. The present paper examines in greater theoretical and experimental detail the role of friction in accounting for the forces of reaction on a fixed ladder. The reported measurements confirm that forces parallel and normal to the support at the top of the ladder are linearly proportional with a constant coefficient of friction irrespective of the magnitude or location of the load, as assumed in the theoretical model. However, measurements of forces parallel and normal to the support at the base of the ladder are linearly proportional with coefficients that depend sensitively on the location (although not the magnitude) of the load. This paper accounts quantitatively for the different effects of friction at the top and base of the ladder under conditions of usual use whereby friction at the vertical support alone is insufficient to keep the ladder from sliding. A theoretical model is also proposed for the unusual circumstance in which friction at the vertical support can keep the ladder from sliding.
\end{abstract}

\section{Keywords}

Forces on a Ladder, Static Equilibrium, Law of Static Friction, Statically Indeterminate, Forces of Reaction

\section{Introduction}

In a recent publication [1] to be referred to as Part I, the author provided a 
theoretical solution and supporting experimental test of the long unresolved problem to determine the four equilibrium forces of reaction on an inextensible ladder [2] inclined against a rough vertical wall, as schematically illustrated in Figure 1. The ladder was modeled as an Euler-Bernoulli [E-B] beam [3] [4] [5] in which the force of friction between the ladder and the wall constituted a critical part of the solution. The objectives of the present paper, designated Part II, are twofold: 1) to demonstrate quantitatively that the forces of reaction measured in Part I are consistent with the law of static friction [6] for the usual prevailing condition that friction at the wall alone cannot keep the ladder from sliding, and 2) to clarify several important points of confusion regarding the differences in the relations governing friction at the wall and at the ground.

\subsection{Background}

Despite its practical importance to workplace safety [7] [8] and personal injury litigation [9], as well as being an archetypical model system in rigid-body mechanics [10] [11] [12], the problem of the reaction forces on a ladder had remained inadequately solved and experimentally untested for much of the past 100 years. The core of the difficulty was that the problem is statically indeterminate if the surface of the vertical support is not frictionless. In such circumstances there are 4 unknown reactions, but only 3 equations of static equilibrium, corresponding to the vanishing of 1) net horizontal force, 2) net vertical force, and 3) net torque about any stationary point. Although the fixed ladder modeled as an E-B beam has routinely served in the pedagogical literature to illustrate the condition of static equilibrium, the worked models were always simplified by neglect of friction at the wall in nearly every mechanics textbook known to the author in use from the early 20th Century [13] to the present time [14]. In the

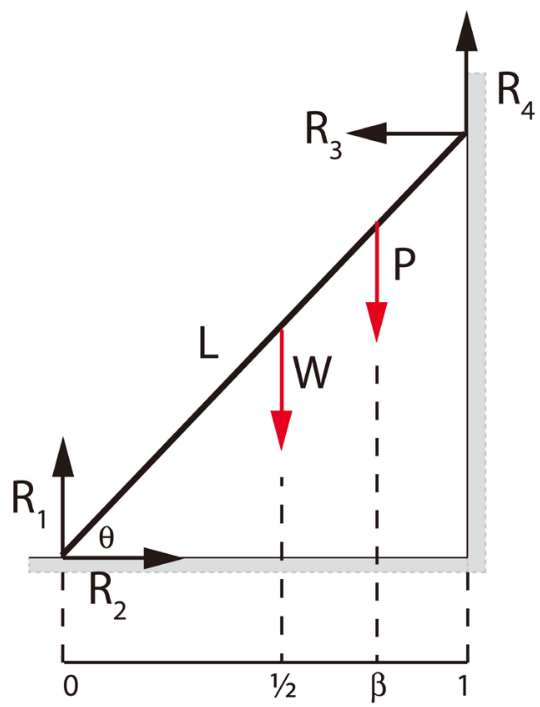

Figure 1. Schematic diagram of the reaction forces $\left(R_{1}, R_{2}, R_{3}, R_{4}\right)$ by the ground and wall on a uniform ladder of length $L$ inclined at angle $\theta$ to the ground. $W$ is the ladder weight acting at the center of mass; $P$ is a load applied at a fraction $\beta$ of the length $L$. 
very few cases where the system included friction at the wall, the problem did not call for, nor provide, expressions for the reactions at static equilibrium [15]. Moreover, the author is unaware of any previously published experimental measurement of these reactions apart from [1].

In general, the solution to a statically indeterminate mechanics problem calls for complementary information ordinarily in the form of a restrictive boundary condition or supplemental external force. Examples of the former, commonly found in textbooks and treatises covering the mechanics of continuous or deformable media, are cases of a statically indeterminate axially or transversely loaded beam [16] [17]. In these examples, the complementary information required to solve for unknown forces or torques depends on the elastic and geometric properties of the beam through the modulus of elasticity $E$, the cross-sectional area $A$, and bending moment of inertia $I$. Correspondingly, several published attempts at solving the ladder problem were based on the assumption that the ladder was subject to axial compression [18] or a bending deformation [19].

In part I the author systematized the different approaches into three distinct fundamental physical models on the basis of the constraints imposed at the ground and wall supports. Models 1 and 2 involved constraints on axial compression and bending deformation. In Model 3, the ladder was free to rotate (but not slide) about the contact point at the ground and to displace vertically against friction at the contact with the wall. The three models were solved analytically, and the predicted forces of reaction of each model were compared with measured forces of reaction as a function of load $P$ and load location (as a fraction $\beta$ of the ladder length $L$ ) measured from the origin at the ground. The experimental reaction forces, measured on both a fixed ladder and on an actual E-B beam, agreed well with predictions of Model 3 and disagreed markedly with predictions of the other models. The theoretical expressions for the reaction forces were therefore found to depend only on the ladder weight $W$, load $P$, angle of inclination $\theta$ with the ground, and coefficient of friction $\mu$ between the ladder or beam and the wall-and not on $E, A$, and $I$.

\subsection{Force of Static Friction on a Ladder or Beam}

The phenomenological force of static friction adopted by the author in Model 3 was based on two principles often referred to as Amontons laws [20], which apply to dry (i.e. non-lubricated) surfaces:

- The maximum force of friction is directly proportional to the applied load.

- The force of friction is independent of the apparent area of contact.

Application of Equation (1) to the contact at the wall related the vertical reaction $R_{4}$ to the horizontal reaction $R_{3}$ by a linear expression

$$
R_{4}=\mu R_{3}
$$

in which the coefficient $\mu$ is a constant depending only on the surfaces of the beam and wall, and not on the angle of inclination of the beam or the magnitude or location of impressed loads. Equation (3) removed the indeterminacy of the 
problem and, together with the 3 equations of static equilibrium, led uniquely to the solution [1]

$$
\begin{gathered}
R_{3}=R_{2}=\frac{R_{4}}{\mu}=\frac{\frac{1}{2} W+\beta P}{\tan \theta+\mu} \\
R_{1}=W+P-\mu R_{3}=\frac{W\left(\tan \theta+\frac{1}{2} \mu\right)+P(\tan \theta+(1-\beta) \mu)}{\tan \theta+\mu}
\end{gathered}
$$

where the first equality in Equation (4) expresses the vanishing of the net horizontal force in static equilibrium, and the first equality in Equation (5) expresses the vanishing of the net vertical force.

The coefficient $\mu$ is not predicted by the model, but is obtained empirically from the data (i.e. the measured reactions) by a method of visual inspection and adjustment [21] or by standard statistical fitting procedures such as the method of maximum likelihood, method of least squares, or Bayes' theorem [22]. As shown in detail in Part I, the forces of reaction given by Equations (3)-(5) for a constant value of $\mu$ satisfactorily accounted for all empirical reactions measured for a wide range of loads $P$ and load locations $\beta$ for both a fixed ladder and a single E-B beam. (The ladder and beam were made of different materials - metal and wood, respectively-and therefore had different frictional constants $\mu$.) Models dependent on the elastic constants of the two structures did not agree with the data.

Agreement between theory (Model 3) and experiment notwithstanding, it is important for both conceptual and practical purposes to show explicitly how well the reactions at the wall satisfy the assumed law of static friction. This demonstration is given in Section 2.

Resolution of the ladder problem by complementary information in the form of the proportionality relation Equation (3) raises an important general question regarding the application of Amontons laws. It is to be understood, of course, that the laws of friction are not physical laws on par with such universal principles as the laws of thermodynamics or the law of conservation of electric charge. Rather, statement (1) is a kind of constitutive relation that depends on the properties of materials and the geometry of their surfaces. Nevertheless, the laws of friction find wide application throughout science and engineering, and it is necessary to be able to apply them correctly.

The question that arises is this: If the forces of reaction parallel and normal to the wall are related by Equation (3), can one likewise relate the forces of reaction parallel $\left(R_{2}\right)$ and normal $\left(R_{1}\right)$ to the ground by a comparable expression

$$
\left[R_{2}=\mu_{g} R_{1}\right]
$$

where $\mu_{g}$ is the corresponding frictional constant and can differ from $\mu$ ? The equation is enclosed in square brackets to emphasize that it is a conjecture to be examined, and not an established equality.

The short answer to the question is "No". Equation (3) and Equation (6) can- 
not both be correct because these 2 independent relations, together with the 3 equations of static equilibrium, constitute 5 independent relations to determine 4 unknown forces. Thus, the system would be over-determined and there would in general be no self-consistent solution. Since the experiments in Part I provided strong evidence in support of Equation (3), it must follow that Equation (6) cannot be correct as it stands. But, given that there is friction at both the wall and ground, why is there asymmetry in the application of the law of static friction, and what is the theoretically valid relation that replaces Equation (6)? These questions are answered in Section 2.

\subsection{Outline of Paper}

In Section 2 the experiments in Part I are briefly recapitulated with additional technical details concerning the measurements of pressure, shear, and strain. The measured forces of reaction at the wall are shown to confirm directly the validity of Equation (3). An exact expression relating the parallel and normal forces of reaction at the ground is derived and tested against experimental results. Issues concerning the apparent asymmetric application of the laws of static friction are resolved.

Conclusions to this work are summarized in Section 3.

\section{Relation between Parallel and Normal Reactions}

\subsection{Experimental Arrangement and Procedure}

Figure 2 shows a schematic diagram of the experimental setup for measurements made on a fixed ladder $(L=244 \mathrm{~cm} ; W=18.9 \mathrm{lbs}=84.07 \mathrm{~N})$ inclined at $\theta=68^{\circ}$ to the ground. Panel A shows a front view and panel B a side view of the ladder resting on two force platforms (one under each rail) level with the ground, with the top of the ladder resting against a third force platform mounted vertically to the wall. A sequence of loads $P$, composed of 1 to 4 lead bricks of weight $25 \mathrm{lbs}(111.21 \mathrm{~N})$ each, was placed at fractional lengths along the ladder given by $\beta=(32,62,92,122,152,182) / 244$.

An analogous setup (not shown) was employed to measure the reactions on a single Euler-Bernoulli wood beam $\left(L_{b}=204 \mathrm{~cm}, W_{b}=15.3 \mathrm{lbs}=67.9 \mathrm{~N}\right)$ inclined at $\theta_{b}=57.7^{\circ}$ to the ground. Loads were applied in units of $2 \mathrm{~kg}$ masses stacked on a weight hanger of $444 \mathrm{~g}$ hooked to eyelets at positions $\beta=(42,72,102,132,162,192) / 204$ along the beam length.

External and internal features of the force platforms are respectively shown in frames A and B of Figure 3. Vertical red arrows in frame A mark the locations of the 4 load cells that register pressure on the platform and therefore reactions normal to the wall or ground. These load cells are indicated by the 4 red circles in frame B. The red double arrow in frame A marks the orientation for measuring shear on the platform and therefore reactions parallel to the wall or ground. This orientation corresponds to the direction of deformation of the central load cell marked by a red double arrow in frame B. 
(A)

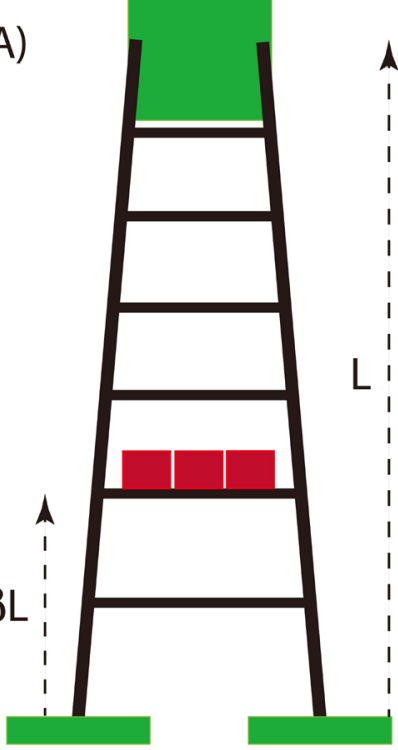$$
1
$$

(1)

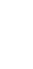

(B)

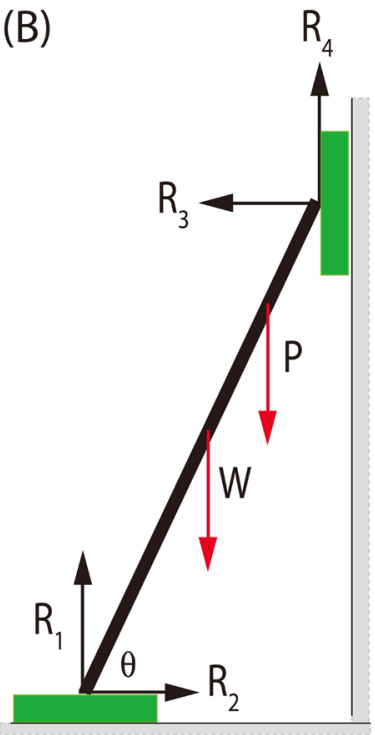

Figure 2. (A) Front view of experimental setup to measure the forces of reaction by the wall and ground on a simple ladder. (B) Side view showing disposition of all forces. Green boxes signify force platforms; red boxes represent lead bricks. The actual ladder used in the experiment had 7 rungs of which 6 were used.

(A)

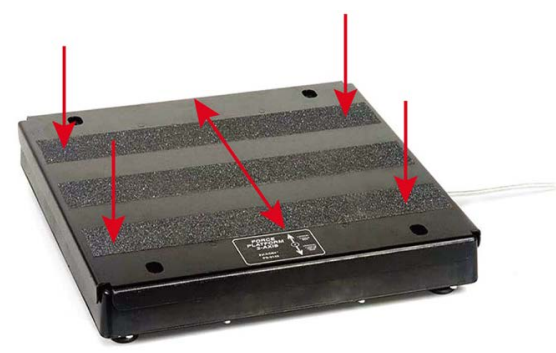

(B)

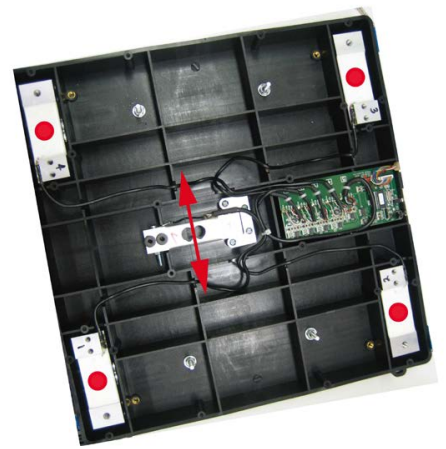

Figure 3. (A) External view of force platform comprising 4 strain gauges (corner arrows) to sense pressure and 1 strain gauge (double arrow) to sense shear. (B) Internal view showing location of pressure gauges (red circles) and shear gauge (red double arrow). The circuit board (green) contains a Wheatstone bridge circuit.

Figure 4 shows an individual load cell in detail. Depending on orientation, the same cell can measure strain due to either pressure or shear. Each load cell comprises 2 parallel strain gauges on opposite side faces. The strain gauge itself consists of a metal foil covered by an insulated flexible support, seen as the white 
(A)

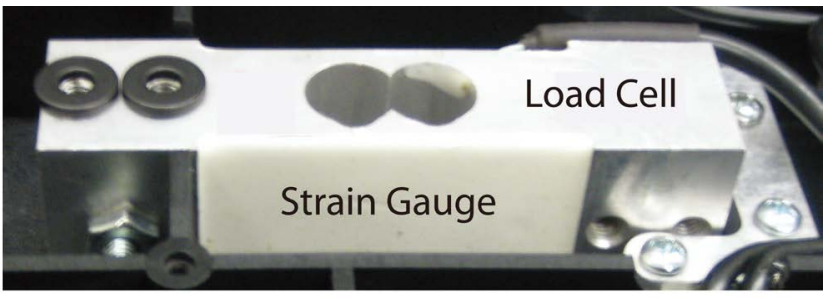

(B)

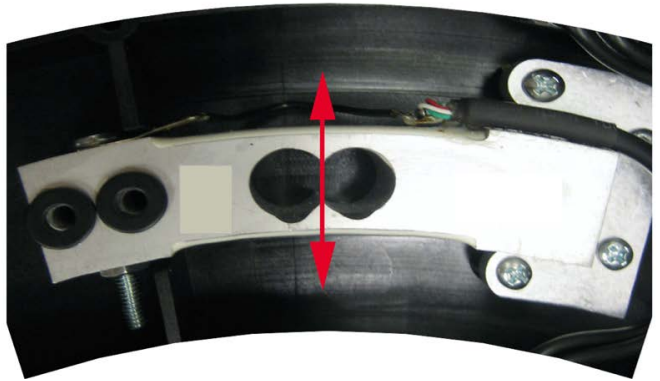

Figure 4. (A) Side view of double-bending beam load cell with strain gauges in front (white rectangle) and rear (not seen). (B) Top view of load cell employed to measure shear, i.e. force parallel to the contact surface of the force platform. The deformation stretches the upper strain gauge and compresses the lower strain gauge (exaggerated for visibility). The difference in resistance of the two gauges, as determined by a Wheatstone bridge circuit, is proportional to the strain.

rectangle on the front face of the load cell in frame A. When a deformation stretches the metal foil, as shown (to an exaggerated extent for visibility) in the top strain gauge of frame $\mathrm{B}$, the resistance of the foil is slightly increased. Correspondingly, when a deformation compresses the metal foil, as shown in the bottom strain gauge of frame $\mathrm{B}$, the resistance of the foil decreases slightly. The degree of strain is proportional to the difference in resistance of the upper and lower strain gauges, as determined by means of a Wheatstone bridge circuit which detects a potential difference between the two foils. As a typical example, a bridge excitation voltage of $10 \mathrm{~V}$ can give rise to a detectable output voltage on the order of millivolts, thereby permitting measurement of strains (length change per initial length) of a few parts in 1000.

\subsection{Test of the Law of Static Friction}

The relation between parallel and normal forces of reaction on a single Euler-Bernoulli wood beam is shown graphically in Figure 5 for the reactions at the ground (panel A) and at the wall (panel B). Each set of 4 circles of a given color mark measurements at a fixed fractional load location $\beta$ for the 4 loads (in newtons) given by $P(k)=(2 k+0.444) g$ for $k=2,3,4,5$ with $g=9.8 \mathrm{~m} \cdot \mathrm{s}^{-2}$. The index $k$ is shown in panel A above the corresponding set of 5 values of $\beta$, which are color coded as follows: $\frac{42}{204}$ (blue), $\frac{72}{204}$ (red), $\frac{102}{204}$ (green), $\frac{132}{204}$ (gold), $\frac{162}{204}$ (brown). The load index $k$ is not needed in panel B since circles of the same color follow an upward progression with load as in panel A. 

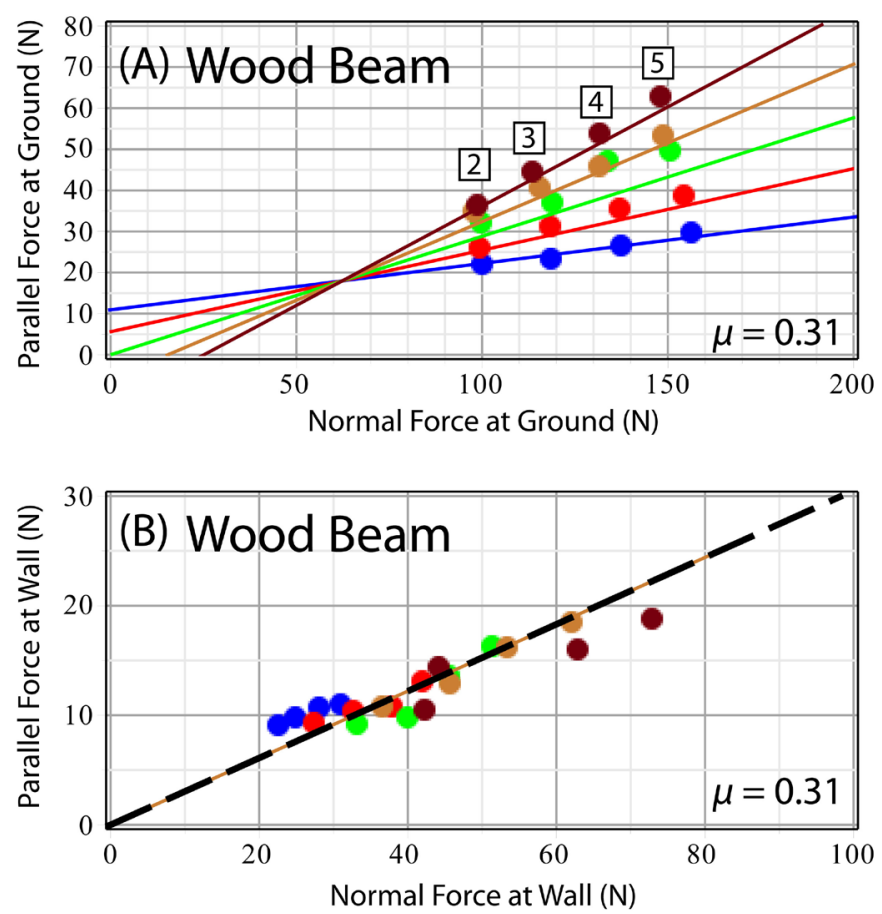

Figure 5. Single wood beam: (A) plot of reaction $R_{2}$ parallel to the ground as a function of reaction $R_{1}$ normal to the ground and (B) plot of reaction $R_{4}$ parallel to the wall as a function of reaction $R_{3}$ normal to the wall, for 4 different loads $P$ at 5 different locations $\beta$ (defined by color). $P=(43.6,63.2,82.8,102.4)$ N. $\beta=\frac{42}{204}$ (blue), $\frac{72}{204}$ (red), $\frac{102}{204}$ (green), $\frac{102}{204}$ (gold), $\frac{162}{204}$ (brown). Measured values are marked by colored circles; solid lines in panel A and the dashed line in panel B show theoretical values calculated from Equations (3)-(5) for wall coefficient $\mu=0.31$. The index $k=2,3,4,5$ gives the load in $\mathrm{N}$ by $P(k)=9.8 \times(2 k+0.444)$. Vertical and horizontal scales are in newtons $(\mathrm{N})$.

The experimental points in panel B are consistent with the assumed law of static friction in which the parallel reaction at the wall is the same linear function of the normal reaction at the wall irrespective of the magnitude and location of the load. The superposed linear function (dashed black line), calculated theoretically from Equation (3) and Equation (4), bears out Amontons 1st law for a frictional coefficient $\mu \approx 0.31$ at the wall. Although there is a narrow spread of points about the dashed line, each set of 4 points of the same color approximates a line of the same slope-and therefore same value of $\mu$-as the theoretical line.

In contrast to the single linear relation in panel $\mathrm{B}$, the pattern of experimental points in panel A shows unambiguously that each set of 4 points of a given color (i.e. variation of $R_{2}$ and $R_{1}$ with $P$ for fixed load location $\beta$ ) follows a linear relation whose slope and therefore frictional coefficient depend on $\beta$. The solid lines in panel A were calculated theoretically from Equation (4) and Equation (5) for the same wall coefficient $\mu \approx 0.31$ as in panel B. Thus, the conjectured Eq- 
uation (6) must actually take the form

$$
R_{2}=\mu_{g}(\beta) R_{1} .
$$

The functional form of $\mu_{g}(\beta)$ can be derived from Equation (4) and Equation (5) by noting that a differential change in load $\mathrm{d} P$ at constant $W$ and $\theta$ results in differential changes in reactions $R_{1}$ and $R_{2}$ of the form

$$
\begin{aligned}
& \mathrm{d} R_{2}=K \beta \mathrm{d} P \\
& \mathrm{~d} R_{1}=K(\tan \theta+\mu(1-\beta)) \mathrm{d} P
\end{aligned}
$$

where $K$ is a constant. It then follows that the slope

$$
\mu_{g}(\beta)=\frac{\mathrm{d} R_{2}}{\mathrm{~d} R_{1}}=\frac{\beta}{\tan \theta+\mu(1-\beta)}
$$

depends on load location $\beta$, but is independent of the magnitude $P$ of the load. Thus, as a consequence of the law of static friction (3) at the wall support, a modified linear force law, Equation (7) with slope given by Equation (9), turns out to be applicable at the ground support.

The variation of $R_{2}$ as a function of $R_{1}$ measured (in newtons) on a fixed ladder is shown in Figure 6 for loads $P=(25,50,75,100) \mathrm{lbs}$ at locations $\beta=\frac{32}{244}$ (blue), $\frac{62}{244}$ (red), $\frac{92}{244}$ (green), $\frac{122}{244}$ (gold), $\frac{152}{244}$ (cyan), $\frac{182}{244}$ (brown). Superposed on each set of 4 points of the same color is both a linear fit (dashed line) obtained by visual trial and adjustment and the theoretical curve (solid line) calculated from Equation (4) and Equation (5). Table 1 records the empirical and theoretical slopes of the lines (calculated from Equation (9)), which are in excellent agreement for all placements of the load except for $\beta=\frac{122}{244}$. The discrepancy in the latter case is slightly greater than for the other load locations $\beta$, both lower and higher along the ladder. The cause is uncertain, but conceivably could have arisen from a small displacement of one or both rails of the ladder from the center of the horizontal force platform (under which is the load cell to measure shear).

As discussed in Part I, the force platforms described in the previous section did not permit direct measurement of the parallel force $R_{4}$ of the wall on the ladder. Unlike a single beam, which could be positioned with the top at the center of the vertical platform-and therefore directly above the load cell that measures shear-the top of the ladder spanned the width of the platform. Small deviations in position of the ladder resulted in erratic variations in the resulting values for $R_{4}$. Nevertheless, $R_{4}$ could be obtained independently from the equation of static equilibrium in the vertical direction: $R_{4}=P+W-R_{1}$. Substitution of Equation (3) for $R_{4}$ leads to the relation

$$
R_{1}+\mu R_{3}=P+W
$$

which provides an independent test of the assumed frictional force law. In Figure 7 the left side of Equation (10) is plotted against the right side as a function 


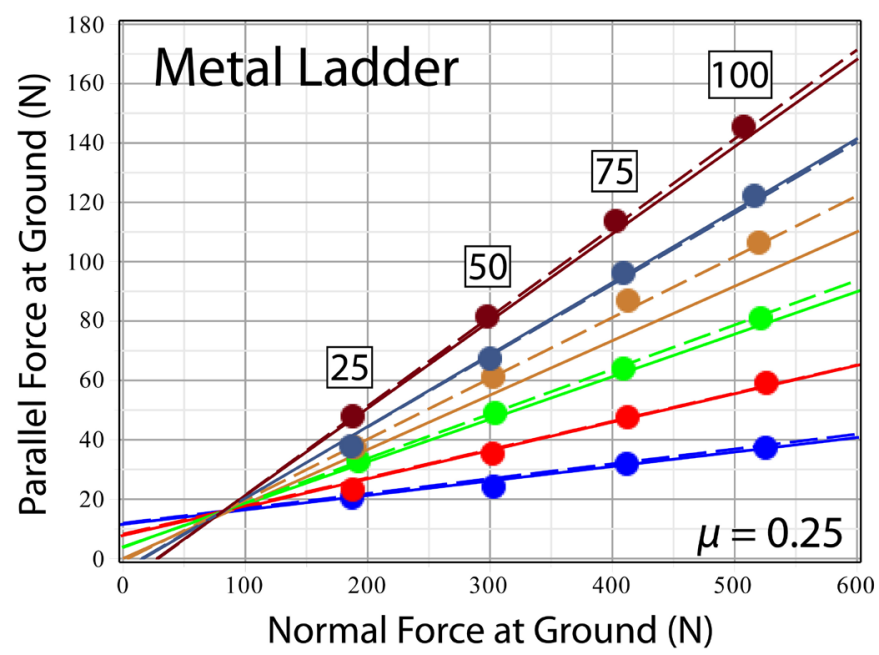

Figure 6. Simple metal ladder: plot of reaction $R_{2}$ parallel to the ground as a function of reaction $R_{1}$ normal to the ground for 4 different loads $P=(25,50,75,100)$ lbs (indicated above each associated column of points) at 6 different locations (defined by color): $\beta=\frac{32}{244}$ (blue), $\frac{62}{244}$ (red), $\frac{92}{244}$ (green), $\frac{122}{244}$ (gold), $\frac{152}{244}$ (cyan), $\frac{182}{244}$ (brown). Vertical and horizontal scales are in newtons $(\mathrm{N})$. Dashed lines are linear fits to the data; solid lines are theoretically calculated values from Equations (3)-(5) for $\mu=0.25$.

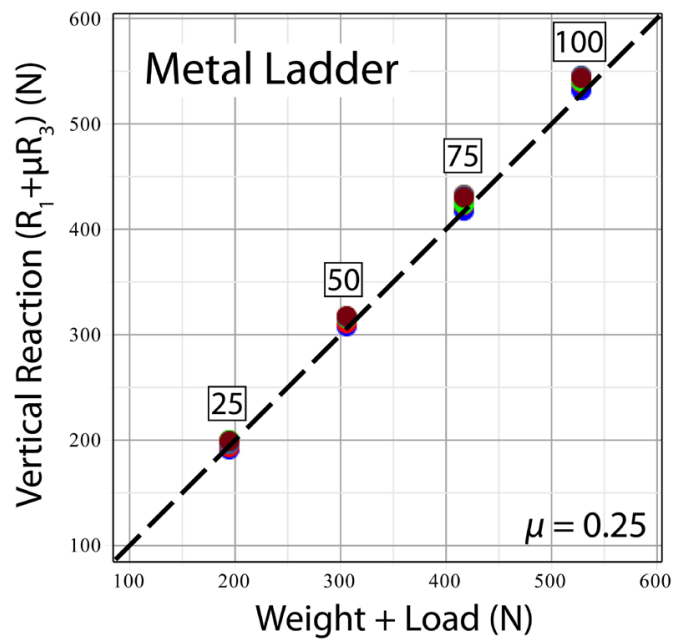

Figure 7. Simple metal ladder: plot of total upward reaction $R_{1}+R_{4}$ against total downward force $W+P$ with implementation of the law of static friction $R_{4}=\mu R_{3}$. The validity of the law requires that all points fall on the theoretical line (dashed) of unit slope irrespective of the magnitude of $P$ (shown in lbs) and the location $\beta$ (color coded as in Figure 6). Vertical and horizontal scales are in newtons (N).

of the 4 loads $P$ for each of the 6 load locations $\beta$. If Equation (3) correctly represents the relation between parallel and normal forces of reaction at the wall, then the resulting plot should be a single straight line of slope 1 upon which all 24 points should closely lie, since $\mu$ is expected to be independent of $P$ and $\beta$. Figure 7 shows that all experimental points do indeed fall on or very near to the theoretical curve (dashed line) calculated from Equations (3)-(5). 
Table 1. Linear Variation of Reaction $R_{2}$ with $R_{1}$ (Ladder parameters: $W=18.9 \mathrm{lbs}, L$ $=244 \mathrm{~cm}, \theta=68^{\circ}$ ).

\begin{tabular}{ccc}
\hline Load Location $\beta$ & Theoretical $\mu_{g}(\beta)$ & Empirical $\mu_{g}(\beta)$ \\
\hline $32 / 244$ & 0.049 & $0.050 \pm 0.005$ \\
$62 / 244$ & 0.095 & $0.095 \pm 0.005$ \\
$92 / 244$ & 0.143 & $0.150 \pm 0.005$ \\
$122 / 244$ & 0.192 & $0.205 \pm 0.005$ \\
$152 / 244$ & 0.242 & $0.240 \pm 0.005$ \\
$182 / 244$ & 0.294 & $0.300 \pm 0.005$ \\
\hline
\end{tabular}

\subsection{Constraint on Sliding at the Ground}

A more complete and accurate formulation of the law of static friction-i.e. the friction between two dry bodies in the absence of relative motion-takes the form of an inequality. Stated in words [6]:

- The force of sliding friction between two surfaces relatively at rest is less than or equal to a certain constant times the normal force to the surfaces.

- The maximum force of friction between the two surfaces, which is the tangential force required to initiate motion, is directly proportional to the normal force.

- The proportionality constant in statement (12) is the defined coefficient of friction.

Applied to the contact of the ladder at the ground, statements (11)-(13) become

$$
R_{2} \leq F_{\max }=\mu_{0} R_{1}
$$

in which $F_{\max }$ is the maximum frictional force that the ground can provide, and $\mu_{0}$ is the coefficient of friction of the ladder with the ground. The equality sign applies at the verge or onset of relative motion of the two surfaces. The physical content of Equation (14) is that if $F_{\max }$ does not exceed the parallel force of reaction $R_{2}$ (which, by the equations of static equilibrium, is equal to the normal force of reaction $R_{3}$ on the ladder by the wall; see Figure 1), then the ladder will slide along the ground.

Substitution of Equation (4) for $R_{2}$ into Equation (14) leads to the inequality

$$
\mu_{0} \geq \frac{\frac{1}{2} W+\beta P}{W\left(\tan \theta+\frac{1}{2} \mu\right)+P(\tan \theta+(1-\beta) \mu)}
$$

which, to insure no sliding, relates the coefficient of friction $\mu_{0}$ at the ground and the frictional constant $\mu$ at the wall. If the wall is frictionless and the ladder carries no load, then Equation (15) reduces to a purely geometric relation

$$
\mu_{0} \geq(2 \tan \theta)^{-1}
$$

that is frequently found in elementary mechanics textbooks [14]. Alternatively, 
one can re-express Equation (15) to yield the threshold angle of inclination below which the ladder will slide

$$
\tan \theta_{\min }=\frac{\frac{1}{2} W\left(1-\mu_{g} \mu\right)+P\left(\beta-\mu_{g} \mu(1-\beta)\right)}{\mu_{g}(W+P)} .
$$

The practical utility of relation (15) or (17) is that it determines the angles of inclination that should be safe for a climber of weight $P$ to ascend to a specified height. Figure 8 illustrates the content of (15) for a $150 \mathrm{lb}$ climber ascending a $25 \mathrm{lb}$ ladder leaning against a wall with frictional constant 0.25 . In the figure, solid curves are plots of the right side of (15) for a fixed value of $\beta$ as a function of angle of inclination. Horizontal dashed lines mark the coefficient of friction at the ground, i.e. values of the left side of relation (15). For given values of $\mu_{0}$ and $\beta$, the ladder should not slide at the ground if inclined at an angle for which the corresponding solid constraint curve is below the corresponding dashed line. For example, if $\mu_{0}=0.3$ (orange dashed line) and the $150 \mathrm{lb}$ climber is to ascend safely halfway up the ladder, $\beta=0.5$ (solid green curve), then the ladder should be set an angle with the ground of $\theta \geq \sim 60^{\circ}$.

\subsection{Correct Use of Static Friction at the Wall and at the Ground}

In view of the content of Section 2.3, the question posed previously regarding the difference in how the laws of friction were applied at the wall and at the ground is seen to be the wrong question. Rather than asking why an equation of the form of (3) does not apply at the ground, the appropriate enquiry should be why an inequality of the form of (14) does not apply at the wall.

The answer to the right question is that use of Equation (3) at the wall is entirely

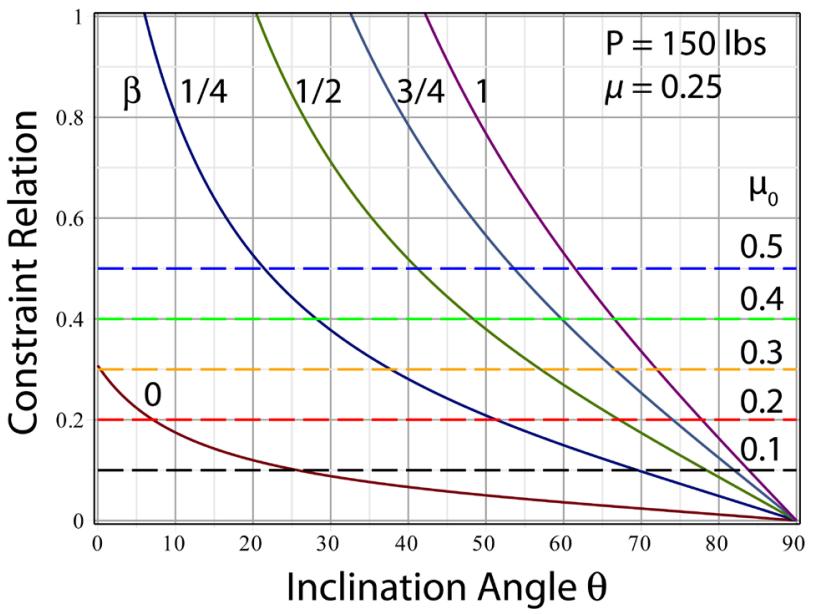

Figure 8. Plot of frictional constraint relation (15) vs angle of inclination (solid curves) for a $150 \mathrm{lb}$ climber ascending a $25 \mathrm{lb}$ ladder to fractional length $\beta=0, \frac{1}{4}, \frac{1}{2}, \frac{3}{4}, 1$. Horizontal dashed lines mark values of the friction coefficient $\mu_{0}=0.5,0.4,0.3,0.2,0.1$. The range of angles for which the ladder does not slide corresponds to lines of specified $\beta$ below the lines of given $\mu_{0}$. 
consistent with the laws of static friction under the prevailing condition by which equilibrium of the ladder or beam was maintained. In the reported experiments, the ladder did not slide along the ground because friction provided by the ground exceeded the normal reaction $R_{3}$ on the ladder by the wall. If the ground were frictionless, the ladder would have slid down the wall because the maximum upward force of friction at the wall was less than the residual downward vertical force $W+P-R_{1}$. Note, however, that the vertical reaction $R_{4}$ on the ladder is the maximum force of friction obtainable from the wall-and therefore, according to statement (12), $R_{4}$ should be directly proportional to the normal force $R_{3}$ at the wall. In other words, Equation (3) - and not an inequality analogous to relation (14)-constitutes a legitimate application of the laws of static friction to the ladder.

It is important to underscore that the theoretical model that was confirmed experimentally in Part I and in the preceding sections of Part II predicts the forces of reaction on a ladder under usual conditions of use. By "usual" is meant that the friction provided by the vertical support (the wall) is insufficient to prevent the ladder from sliding at the ground if the contact between the ladder and the ground were smooth (i.e. nearly frictionless). One can imagine, however, a system-such as a ladder inclined against a very rough stone wall-whereby the maximum force of friction at the wall exceeds the net vertical force downward. Then, if it is still the case that the maximum force of friction at the ground exceeds the parallel reaction $R_{2}$, the laws of static friction become inequalities at both the wall and ground. Such a statically indeterminate system would again be unsolvable without further complementary information because the forces of friction cannot be related to the forces of reaction. Although the coefficients of friction at the wall and ground would determine whether the ladder slid or not, they would not enter, and therefore could not determine, the mathematical expressions for the forces of reaction in static equilibrium.

The author is unaware of any published theory or experiment that examined the static equilibrium of a ladder supported at both base and top by surfaces sufficiently rough that each alone was capable of preventing the ladder from sliding. One approach to analyzing such a system within the framework of boundary constraints introduced in Part I, might be to regard the ladder (or E-B beam) as pinned at both the ground and wall. To recapitulate briefly, the three fundamental kinds of supports commonly encountered in the mechanics of deformable media are roller, pin, and fixed [23]. The boundary conditions defining a pinned contact point is that (a) translational displacement at that point is zero; (b) the bending moment (torque) at that point is zero; (c) the deformation curve at that point has a non-zero slope. Conditions (b) and (c) reflect the fact that the beam (or a segment of a deformable beam) can rotate about the pinned contact.

The model of a ladder as a single E-B beam pinned at both ends was not included among the 3 models analyzed in Part I because it led to results that could be dismissed immediately as unrepresentative of the statics of a ladder in usual 
use. These results, however, may apply to the extraordinary condition discussed now, and therefore the analysis is given here. The steps of the analysis are as follows.

1) Transform reactions $\left(R_{1}, R_{2}, R_{3}, R_{4}\right)$, which are perpendicular or parallel to the ground or wall, into reactions $\left(A_{1}, A_{2}, B_{1}, B_{2}\right)$, which are axial or transverse with respect to the beam, as shown in Figure 9.

2) Use the equations of static equilibrium to solve for the transverse reactions $\left(B_{1}, B_{2}\right)$. This can be done because there is no unknown internal bending moments at pinned contacts with the ground and wall. (Note: Such moments exist for a fixed contact. See [1].)

3) Write the expression for the axial strain energy. This will contain one of the axial reactions. (In the analysis below, the axial strain energy is expressed in terms of $A_{2}$.)

4) Express the axial reaction in terms of one of the perpendicular or parallel reactions. (In the analysis below, axial reaction $A_{2}$ is expressed in terms of perpendicular reaction $R_{1}$.)

5) Use Castigliano's theorem [17] (pp 201-217) to implement the boundary condition that the end points of the ladder cannot displace either perpendicular or parallel to the ground or wall. Solve the resulting equation for the perpendicular or parallel reaction. (The analysis below applies the constraint on perpendicular displacement at the ground and solves for $R_{1}$.)

6) From steps 2 and 5 the entire set of reactions $\left(R_{1}, R_{2}, R_{3}, R_{4}\right)$ can be obtained.

From Figure 9 one can relate the forces normal and parallel to the ladder to the forces axial or transverse to the ladder as follows:

$$
\begin{aligned}
& A_{1}=R_{1} \sin \theta+R_{2} \cos \theta \\
& B_{1}=R_{1} \cos \theta-R_{2} \sin \theta \\
& A_{2}=-R_{3} \cos \theta+R_{4} \sin \theta \\
& B_{2}=R_{3} \sin \theta+R_{4} \cos \theta
\end{aligned}
$$

with inverse relations

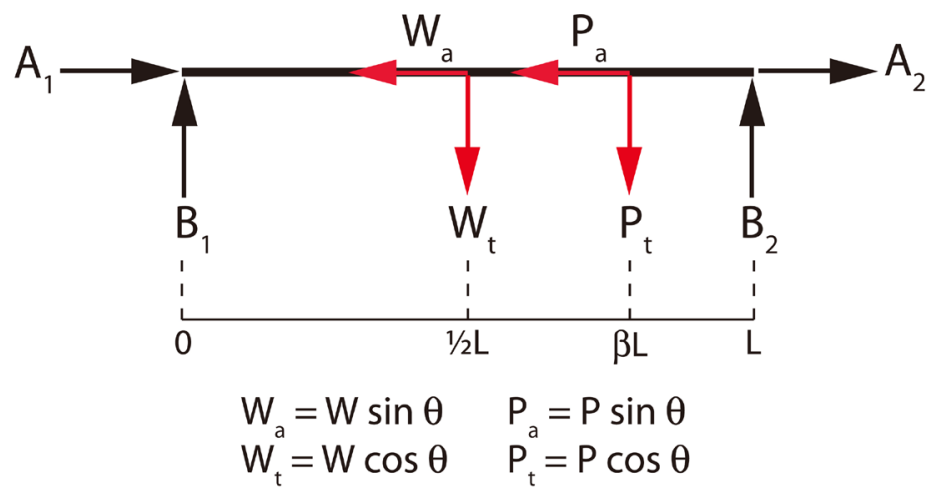

Figure 9. Decomposition of forces of reaction into components axial (a) and tangential (t) with respect to the beam. $W$ and $P$ are respectively the beam weight and impressed load; $\theta$ is the angle of inclination of the beam with respect to the ground. 


$$
\begin{aligned}
& R_{1}=B_{1} \cos \theta+A_{1} \sin \theta \\
& R_{2}=-B_{1} \sin \theta+A_{2} \cos \theta \\
& R_{3}=-B_{2} \sin \theta-A_{2} \cos \theta \\
& R_{4}=B_{2} \cos \theta+A_{2} \sin \theta
\end{aligned}
$$

Solution of the equations of static equilibrium for the transverse reactions lead directly to

$$
\begin{aligned}
& B_{1}=\left(\frac{1}{2} W+(1-\beta) P\right) \cos \theta \\
& B_{2}=\left(\frac{1}{2} W+\beta P\right) \cos \theta
\end{aligned}
$$

From Equation (20) and the second and fourth relations of Equation (18), one can express $R_{3}$ and $R_{4}$ in terms of $R_{1}$

$$
\begin{aligned}
& R_{3}=\left(R_{1}-\frac{1}{2} W-(1-\beta) P\right) \frac{\cos \theta}{\sin \theta} \\
& R_{4}=W+P-R_{1}
\end{aligned}
$$

Substitution of Equation (21) into the third relation of Equation (18) leads to the axial reaction $A_{2}$ in terms of the perpendicular reaction $R_{1}$

$$
A_{2}=\frac{W\left(1-\frac{1}{2} \cos ^{2} \theta\right)+P\left(1-\beta \cos ^{2} \theta\right)-R_{1}}{\sin \theta} .
$$

The axial strain energy takes the general form

$$
U_{a}=\frac{1}{2 E A} \int_{0}^{L} F_{a}(x)^{2} \mathrm{~d} x
$$

where $F_{a}(x)$ is the compressive or tensile force throughout the beam. Applying Equation (23) to a ladder modeled as an E-B beam, one finds

$U_{a}=\frac{L}{2 E A}\left[\int_{0}^{\beta}\left(A_{2}-W(1-\xi) \sin \theta-P \sin \theta\right)^{2} \mathrm{~d} \xi+\int_{\beta}^{1}\left(A_{2}-W(1-\xi) \sin \theta\right)^{2} \mathrm{~d} \xi\right]$

after making a transformation of variables $\xi=x / L$. Substitution of Equation (22) into Equation (24) and solution of the equation resulting from Castigliano's theorem

$$
\delta_{\perp}^{(g)}=\frac{\partial U_{a}}{\partial R_{1}}=0,
$$

where $\delta_{\perp}^{(g)}$ is displacement of the contact point at the ground in the direction of force $R_{1}$, leads to

$$
R_{1}=\frac{1}{2} W+(1-\beta) P
$$

from which follows from Equation (21)

$$
R_{4}=\frac{1}{2} W+\beta P
$$

and 


$$
R_{2}=R_{3}=0 .
$$

The axial reactions at the base and top of the ladder

$$
\begin{aligned}
& A_{1}=\left(\frac{1}{2} W+(1-\beta) P\right) \sin \theta \\
& A_{2}=\left(\frac{1}{2} W+\beta P\right) \sin \theta
\end{aligned}
$$

follow from Equation (28) and the first and third relations of Equation (18).

The set of reactions (26)-(28) are independent of the angle of inclination $\theta$, in marked contrast to the forces of reaction on an inclined ladder under usual circumstances. The foregoing solution is likewise obtained if the axial strain energy function (23) were expressed in terms of the parallel reaction $R_{2}$ and the application of Castigliano's theorem took the form

$$
\delta_{\|}^{(g)}=\frac{\partial U_{a}}{\partial R_{2}}=0
$$

where $\delta_{\|}^{(g)}$ is displacement of the ground contact point in the direction of $R_{2}$. In fact, the same solution is obtained by applying Castigliano's theorem directly to the axial strain energy function (24) without making the transformation (22). Although this would have been a simpler way to solve the problem, the approach was not followed here because the corresponding virtual displacement $\delta_{\text {axial }}^{\text {(wall) }}=\partial U_{a} / \partial A_{2}$ or $\delta_{\text {axial }}^{\text {(ground) }}=\partial U_{a} / \partial A_{1}$ would be in a direction not permitted by the ground and wall constraints.

\section{Conclusions}

In the paper [1] designated Part I, the author derived theoretically and confirmed experimentally a solution to the long-standing "ladder problem", i.e. the problem to predict the 4 forces of reaction on a fixed ladder resting on horizontal ground and inclined against a vertical wall. The solution was obtained by combining the equations of static equilibrium and an equation for the force of static friction at the wall. In the present paper, designated Part II, the frictional force measured at the wall was shown to be proportional to the normal force measured at the wall, thereby confirming use of Amontons law of static friction. This relation held true experimentally for the same proportionality constant $\mu$ over a wide range of loads and different load positions along the ladder. Comparable experimental and theoretical agreement was also demonstrated for the 4 forces of reaction measured on a single wood beam inclined against a wall.

The foregoing proportionality of parallel and normal reactions was shown not to hold at the ground. At the ground, the theory predicted-and the measured reactions confirmed-that the force of friction and the normal force were linearly related by a coefficient that depended sensitively on the position (although not the magnitude) of the load. This apparently asymmetric consequence of friction at the wall and at the ground was resolved by noting that, in general, the law of static friction takes the form of an inequality. The law becomes a li- 
near proportionality only when the frictional force is the maximum obtainable from the immovable surface. This explanation envisions a system, such as examined experimentally in this paper and in Part I, in which a movable object (e.g. ladder) can slide against an immovable object (e.g. wall). It is understood, of course, that the force of friction depends on both surfaces.

If this explanation is correct, then the following conclusions can be drawn from it regarding the role of friction in the experiments reported in Parts I and II:

- Friction at the ground physically maintains the ladder or beam in static equilibrium by preventing sliding.

- Friction at the wall (together with the equations of static equilibrium) determines theoretically the four forces of reaction. In other words, the coefficient of friction at the ground does not enter any of the theoretical expressions for the reactions.

- The reason why friction affected the ladder or beam differently at the ground than at the wall (i.e. "inequality" vs. "proportionality") is due to the fact that the magnitude of friction, relative to other acting forces, was different at those two supports. At the ground, the maximum obtainable friction was greater than the parallel reaction $R_{2}$. At the wall, the maximum obtainable friction (identical to the parallel reaction $R_{4}$ ) was less than the net downward force.

- In light of the preceding comments, the frictional constant $\mu$ in Equation (3) is the canonically defined coefficient of friction at the wall-and therefore should yield (within experimental error) the same numerical value independent of load and load placement whether deduced from the forces of reaction on the ladder or measured by any of the standard methods for determining coefficients of static friction [24] [25].

The forces of reaction derived and tested in Parts I and II pertained to a physical system in which the force of friction at the wall was insufficient by itself to maintain the ladder in static equilibrium, i.e. to keep the ladder from sliding if the ground surface was smooth. Under the uncommon circumstance where a ladder is supported by two rough surfaces (ground and wall) at which neither the top nor base of the ladder is on the verge of sliding, the effect of friction at each surface must be expressed by an inequality. This statically indeterminate problem would then require additional complementary information to be solvable. In the model analyzed in Section 2.4 of a single-beam ladder with both ends pinned, this complementary information made use of the axial strain energy function of the beam, although the resulting forces of reaction were found not to depend on the elastic constant $E$.

The author is unaware of any experimental test of such a system. Whether the model of an inclined Euler-Bernoulli beam pinned at both ends satisfactorily describes a ladder in static equilibrium under the hypothetical condition portrayed above can be decided only by an experimental test. Such a test is outside the scope of this paper. 


\section{Acknowledgements}

E. Donovan, W. Emery, J. Hallquist, W. Scully, L. Sewanan, and W. Strange each helped take data at some point during the initial phase of this project. The author also thanks Trinity College for partial support through the research fund associated with the George A. Jarvis Chair of Physics.

\section{Conflicts of Interest}

The author declares no conflicts of interest regarding the publication of this paper.

\section{References}

[1] Silverman, M.P. (2018) Reaction Forces on a Fixed Ladder in Static Equilibrium: Analysis and Definitive Experimental Test of the Ladder Problem, World Journal of Mechanics, 8, 311-342.

[2] For a Description of Ladder Types, See: Ladders 101. http://www.americanladderinstitute.org/page/Ladders101

[3] Love, A.E.H. (1944) A Treatise on the Mathematical Theory of Elasticity. Dover, New York City, NY, 365-370.

[4] Stippes, M., Wempner, G., Stern, M. and Beckett, R. (1961) The Mechanics of Deformable Bodies. C. E. Merrill Books, Columbus OH, USA, 237-244.

[5] https://en.wikipedia.org/wiki/Euler\%E2\%80\%93Bernoulli_beam_theory

[6] Den Hartog, J.P. (1948) Mechanics. Dover, New York City, NY, 83-86.

[7] Hakkinen, K.K., Pesonen, J. and Rajamaki, E. (1988) Experiments on Safety in the Use of Portable Ladders. Journal of Occupational Accidents, 10, 1-19. https://doi.org/10.1016/0376-6349(88)90002-8

[8] Lee, Y.H. and Tung, E.K. (1992) Body and Ladder Mechanical Stresses Analysis in a Climbing Strike. In: Kumar, S., Ed., Advances in Industrial Ergonomics and Safety $I V$, Taylor and French, 1007-1014.

[9] Silverman, M.P. (2009) Final Report: Force of a Ladder on a Railing under Static and Dynamic Conditions, Case of Shattuck v. Wynfield, Hartford Superior Court, Hartford CT, USA.

[10] Moore, E.N. (1983) Theoretical Mechanics. John Wiley \& Sons, New York City, NY, 24-25.

[11] French, A.P. (1971) Newtonian Mechanics. W. W. Norton, New York City, NY, 135.

[12] MacMillan, W.D. (1936) Dynamics of Rigid Bodies. Dover, New York City, NY, 160-161.

[13] Lindsay, R.B. (1933) Physical Mechanics. D. van Nostrand, New York City, NY, 158-163.

[14] Knight, R.A. (2017) Physics for Scientists and Engineers. 4th Edition, Pearson, Boston MA, 313.

[15] Roberts, A.P. (2003) Statics and Dynamics with Background Mathematics. Cambridge University Press, Cambridge UK, 102-103. https://doi.org/10.1017/CBO9780511815812

[16] Hibbeler, R.C. (1997) Mechanics of Materials. Prentice Hall, Upper Saddle River, 137-144, 628-631. 
[17] Budynas, R.G. (1977) Advanced Strength and Applied Stress Analysis. McGraw-Hill, New York, 197-201, 221-232.

[18] Mendelson, K.S. (1994) Statics of a Ladder Leaning against a Rough Wall. American Journal of Physics, 63, 148-150. https://doi.org/10.1119/1.17972

[19] Gonzalez, A.G. and Gratton, J. (1996) Reaction Forces on a Ladder Leaning against a Rough Wall. American Journal of Physics, 64, 1001-1005. https://doi.org/10.1119/1.18317

[20] Bhushan, B. (2002), Introduction to Tribology. John Wiley \& Sons, New York, 207-215.

[21] Silverman, M.P. and Farrah, J. (2018) Bending of a Tapered Rod: Modern Application and Experimental Test of Elastica Theory. World Journal of Mechanics, 8, 272-300. https://doi.org/10.4236/wjm.2018.87022

[22] Silverman, M.P. (2014) A Certain Uncertainty: Nature's Random Ways. Cambridge University Press, Cambridge, 54-83. https://doi.org/10.1017/CBO9781139507370

[23] Hibbeler, R.C. (1997) Mechanics of Materials. 3rd Edition, Prentice Hall, Upper Saddle River, 582-583.

[24] Blau, P.J. (2009) Friction Science and Technology: From Concepts to Applications. CRC Press, Boca Raton, 43-118.

[25] How to Measure Friction. http://www.tribology-abc.com/abc/friction.htm 